\title{
Striking Regularity in Meson and Baryon Production Rates in $e^{+} e^{-}$ annihilations
}

\author{
P. V. Chliapnikov and V. A. Uvarov \\ Institute of High Energy Physics \\ 142284, Protvino, Russia
}

\begin{abstract}
It is shown that $e^{+} e^{-}$data on the production rates of all particles (apart from pions) belonging to the $\mathrm{SU}(3)$ nonets of the pseudoscalar and vector mesons and, to the octet and decuplet of the baryons are determined only by particle spins, isospins and their masses. Contrary to a general belief and popular models, such as the Jetset model, the $2 J+1$ spin counting factor works perfectly. The meson and baryon production rates at $Z^{0}$ pole at LEP as a function of particle mass squared lie on the one universal curve described by a simple formula $<N($ particle $)\rangle=\frac{2 . J+1}{2 I_{m}+1} a \exp \left(-b M^{2}\right)$ with parameters $a=11.28 \pm 0.31$ and $b=3.872 \pm 0.027\left(\mathrm{GeV} / \mathrm{c}^{2}\right)^{-2}$ and $\chi^{2} / N D F=15 / 10$, where $I_{m}$ is isospin for baryons or modified isospin $I_{m}$ for mesons introduced in this paper. Similar universal dependence with the same value of the slope $b$ is observed at PETRA and PEP energies.
\end{abstract}


Hadron production in $Z^{0}$ decays proceeds through two main steps: parton shower development from the primary $q \bar{q}$ pair produced from the $Z^{0}$, followed by fragmentation of the coloured partons into colorless hadrons. The first is well described by QCD, the theory of strong interactions. However, perturbative QCD is not applicable to the soft processes of hadronization. The formation of hadrons, mesons and baryons, out of quarks and gluons has been studied in many experiments in an attempt to understand the hadronization process better and to test phenomenological models of parton fragmentation. The most successful of these models are the string [1] and the cluster fragmentation [2] models implemented in the widely used Monte Carlo programs Jetset 7.4 PS [3] and Herwig 5.8 [4] respectively. However, the number of parameters in these models is quite large. Thus in the Jetset model many parameters control production of pseudoscalar and vector mesons. The most important are the suppression of $s$ quark pair production compared with $u$ or $d$ pair production, the probability that a light meson (containing $u$ and $d$ quarks) has spin 1 and the probability that a strange meson has spin 1.

The treatment of baryon production in $e^{+} e^{-}$annihilations by the existing hadronization models is based on the experimental observations at PETRA and PEP energies that the differential momentum spectra of mesons and baryons are rather similar. This justifies the diquark model in which the fragmentation functions for baryons and mesons are assumed to be almost the same, quark-antiquark and diquark-antidiquark pairs are produced from the sea and a diquark combines with a quark to form a baryon. The relative production rates of the different octet and decuplet baryons are again controlled by free parameters tuned to the experimental data. The number of such parameters is also large. Thus in the Jetset model, the parameters relevant to octet and decuplet baryon production include the diquark to quark ratio $(q q / q)$, the strange to non-strange quark ratio $((s / d)$, the same as used for the mesons $)$, the strange diquark suppression factor $(u s / u d) \cdot(d / s)$, the spin 1 diquark parameter $\frac{1}{3}\left(q q_{1} / q q_{0}\right)$ and the popcorn parameter (see below).

Clearly, with such a number of free parameters, many of which are correlated, the predictive power of these models, especially for baryon production, is very limited and precise measurements of many baryon species are necessary for a proper tuning of the various diquark parameters.

Some deeper understanding of the meson and baryon production mechanisms may come from comparison of the data with a simple ansatz of the Lund string model developed at UCLA [5], which is closely related to the soft QCD concepts of area law behaviour and longitudinal phase space. This model has only 5 major parameters, three of them being used to tune the parton shower and to parameterize the Lund symmetric fragmentation function, one to account for local $p_{T}$ compensation and one for popcorn suppression.

May be new more precise data will shed new light on hadroproduction mechanisms in $e^{+} e^{-}$annihilations. Previous measurements of meson and baryon production in $e^{+} e^{-}$ annihilations at energies below the $Z^{0}$ pole, with few exceptions, suffered from poor statistical precision. More precise information is expected from the LEP experiments (with about $6,000,000$ hadronic $Z^{0}$ decays expected to be accumulated in each of them by the end of the LEP 100 program). But even now the amount of accumulated information is quite impressive.

Therefore in this letter an attempt is made to find some empirical regularities in the meson and baryon production mechanisms using the results of the LEP experiments on the production rates of pseudoscalar and vector mesons and, of octet and decuplet baryons. This attempt was motivated by the following: 
1. The present hadronization models fail to describe all existing LEP data on meson and baryon production.

2. The models use a large number of adjustable parameters, especially in the Jetset model. It is difficult to understand the physical meaning of some of them, such as the strange diquark suppression factor in addition to the usual suppression factor $s / d$.

3. There is a puzzling problem with the $(2 J+1)$ spin counting factor. In the Jetset model, for example, the probabilities that mesons composed from $(u$ or $d), s,(c$ or $b)$ quarks have spin 1 are different and equal to $0.5,0.6$ and 0.75 . The suppression of spin 1 diquarks compared with spin 0 is even larger: the ratio of the corresponding probabilities $\frac{1}{3}\left(u d_{1} / u d_{0}\right)=0.05$ (excluding the factor 3 coming from spin counting).

4. The universality in energy dependence of the average charged particle multiplicities in $e^{+} e^{-}$annihilation and $(p / \bar{p}) p$ collisions $[6]$ imply that the hadron production rates are dominantly determined by some quite simple characteristics such as longitudinal phase space and available energy, the hadron masses, isospins and spins.

We first start with an analysis of the recent results on the production rates of SU(3) octet and decuplet baryons obtained in the LEP experiments. We then compare these with the LEP results on the production rates of pseudoscalar and vector mesons from SU(3) nonets. Finally we repeat this analysis using data at PETRA and PEP energies.

The LEP experiments have presented analyses of the inclusive production of various baryons ${ }^{\dagger}$ including $\Lambda$ [7]-[12], $\Sigma^{+}[13], \Sigma^{+}(1385)$ [8]-[12], $\Xi^{-}[7]-[12], \Xi^{0}(1530)$ [8]-[12], $\Omega^{-}$ $[9,10]$ and protons $[14,15]$ (for a review, see [16]). Measurements of the strangeness or the baryon number compensation mechanism in jets, essential for understanding the popcorn mechanism [17], are reported in $[8,12,18,19]$. The measured overall yields of the SU(3) octet and decuplet baryons are summarized in Table 1, together with the corresponding predictions of the Jetset, Herwig and UCLA models with default parameters.

One can see that in spite of the large number of adjustable parameters, the description of the experimental results on baryon production by Jetset is far from perfect, especially for the decuplet baryons. Herwig, with fewer free parameters, grossly overestimates the $\Xi^{-}$and decuplet baryon production rates. The UCLA model, with still fewer free parameters, surprisingly agrees with the data as well as Jetset.

In comparing the production rates of the different particles one usually uses the measured values corresponding to certain isospin projections. This can be quite misleading. The states with different isospin projections are expected to be produced in $e^{+} e^{-}$annihilations with practically the same probabilities. Therefore for comparison of the production rates of octet and decuplet baryons, the measured production rate for the state with the isospin projection given in Table 1 is multiplied by the $(2 I+1)$ isospin counting factor, assuming that the production rate of the state with unmeasured isospin projection(s) is equal to the measured one. In fact, whenever possible, as in cases of the $\Sigma$ and, later on, of the pions, the kaons and the $K^{*}(890)$, the experimental values of the production rates corresponding to the states with different isospin projections were used to calculate the overall production rate for the particles with the given isospin. However, the $(2 I+1)$ notation will be used even in such cases for clarity.

The octet and decuplet baryon production rates thus calculated are presented in Fig.1 as a function of baryon mass $(M)$ squared $^{\ddagger}$. The predictions of the Jetset, Herwig and UCLA models are also shown in Fig. 1 by the lines joining the predicted rate values for different baryons.

\footnotetext{
† In this paper, unless otherwise stated, antiparticles are implicitly excluded and particles with opposite charge are treated separately. In the experimental papers, the production cross sections for particle and antiparticle and, for the particles with opposite charge are usually combined.

† The particle mass squared was taken at the mean value of the masses squared corresponding to the different isospin projections taken from the PDG tables [20].
} 
An exponential decrease of the octet and decuplet baryon production rates with $M^{2}$ is observed. It explains why the predictions of the UCLA model with only 5 major parameters are on the same level of agreement with the data as the Jetset model: an exponential $\exp \left(-b M^{2}\right)$ mass dependence has been explicitly used in their phenomenology $[5]$.

The fit by the form $a \exp \left(-b M^{2}\right)$ (straight lines in Fig. 1) yields: $a=32.5 \pm 5.1$ and $b=4.15 \pm 0.12\left(\mathrm{GeV} / c^{2}\right)^{-2}$ with $\chi^{2} / N D F=4.5 / 6$ for the octet baryons and, $a=18.3 \pm 11.3$ and $b=3.38 \pm 0.29\left(\mathrm{GeV} / c^{2}\right)^{-2}$ with $\chi^{2} / N D F=0.4 / 3$ for the decuplet baryons. The exponential slopes $b$ for octet and decuplet baryons are the same within 2.5 standard deviations. Another interesting observation is that the difference between the fitted lines for octet and decuplet baryons at $M^{2}=1.8\left(\mathrm{GeV} / c^{2}\right)^{2}$ is around 2 (see Fig. 1 ), suggesting that it can be explained by the $2 J+1$ spin counting factor. If this factor works, then it may well be that the small difference in the slopes $b$ for octet and decuplet baryons is due to statistical and systematic errors of the experimental points. In order to check this, one can compare the octet and decuplet baryon production rates taking them with the $1 /(2 J+1)$ weight factor (i.e. dividing the production rates for octet and decuplet baryons by 2 and 4 respectively). The corresponding results are presented in Fig. 2.

It shows that indeed all data points for octet and decuplet baryons lie on one universal line. The fit by the exponential $a \exp \left(-b M^{2}\right)$ (straight line in Fig. 2) gives

$$
a=12.2 \pm 1.4 \quad \text { and } \quad b=3.92 \pm 0.08\left(\mathrm{GeV} / c^{2}\right)^{-2}
$$

with $\chi^{2} / N D F=14.7 / 11$.

This remarkable fact implies that the spin $2 J+1$ counting factor indeed works and that the production rates of different octet and decuplet baryons are dominantly determined by their masses, isospins and spins. This phenomenon was not observed before because it was hidden by the difference in masses of the different baryons and because the baryon production rates were considered for the separate isospin projections.

After this observation we were unable to resist a temptation to check if this is also true for the mesons belonging to the $\mathrm{SU}(3)$ pseudoscalar and vector meson nonets.

The overall yields of the pseudoscalar and vector mesons from the first two SU(3) nonets measured in the LEP experiments are given in Table 1 . The production rates of the $\eta$ and $\eta^{\prime}$ given in Table 1 are obtained by us from the measured values of < $N(\eta)\rangle=0.298 \pm 0.031$ and $\left\langle N\left(\eta^{\prime}\right)\right\rangle=0.068 \pm 0.024$ for $x_{E} \geq 0.1[26]$ and,$\langle N(\eta)\rangle=$ $0.70 \pm 0.08$ for $0.02 \leq x_{p} \leq 0.3$ [11]. Extrapolation to the full $x$-range is made assuming the unmeasured $x$-regions are represented by the normalized Jetset and Herwig models. The values obtained using these two models are then averaged and the difference between the results of the extrapolations is added to the errors in quadrature.

The corresponding predictions of the Jetset, Herwig and UCLA models for the pseudoscalar and vector meson nonets are also presented in Table 1 . The Jetset model is in reasonable agreement with the data for the pseudoscalar meson nonet, apart from $\eta^{\prime}$, but overestimates the vector meson yields and especially the $\phi$ production rate. The Herwig and UCLA models describe better the general trend of the data although some problems also exist.

In comparing the meson and baryon production rates, the $(2 I+1) /(2 J+1)$ weight factor used for the baryons can not be applied blindly. The two following cases need special care:

1. The $\mathrm{K}^{+}$and $\mathrm{K}^{0}\left(\mathrm{~K}^{*+}(890)\right.$ and, $\left.\mathrm{K}^{* 0}(890)\right)$ and their antiparticles $\mathrm{K}^{-}$and $\bar{K}^{0}$ ( $\mathrm{K}^{*-}(890)$ and $\left.\bar{K}^{* 0}(890)\right)$ belong to the two different isodoublets with the different strangeness $(S=1$ and $S=-1$ ), while the antiparticles of the $\pi$ and $\rho$ belong to the same 
isotriplet. Besides, the strange and non-strange mesons with their antiparticles belong to the same $\mathrm{SU}(3)$ multiplet, which is not true for the baryons and antibaryons. Anyhow since there are four $\mathrm{K}$ meson states $\mathrm{K}^{+}, \mathrm{K}^{0}, \bar{K}^{0}$ and $\mathrm{K}^{-}\left(\mathrm{K}^{*+}(890), \mathrm{K}^{* 0}(890), \bar{K}^{* 0}(890)\right.$ and $\left.K^{*-}(890)\right)$, then for each of them the isospin counting factor equals $2(2 I+1)$.

2. The $I=0$ member of the ground-state pseudoscalar octet mixes with the corresponding pseudoscalar singlet to produce the $\eta$ and $\eta^{\prime}$. Similarly the mixing in the ground-state vector octet with the vector singlet produces the $\omega$ and $\phi$. Usually the physical states $\eta$ and $\eta^{\prime}(\omega$ and $\phi)$ are given in terms of a mixing angle $\theta_{P}\left(\theta_{V}\right)$ and of the octet $\eta_{8}\left(\omega_{8}\right)$ and singlet $\eta_{1}\left(\omega_{1}\right)$ states. But the masses of the SU(3) singlet ground-states are not known and therefore the proper treatment of the $\eta$ and $\eta^{\prime}$ ( $\omega$ and $\phi$ ) production rates in the context of this study is not obvious.

This problem can be solved by introducing the new basic states $\eta_{a}$ and $\eta_{b}\left(\omega_{a}\right.$ and $\left.\omega_{b}\right)$ with the modified mixing angle $\theta_{P m}=45^{\circ}$, so that:

$$
\begin{aligned}
\eta & =\frac{1}{\sqrt{2}} \eta_{a}-\frac{1}{\sqrt{2}} \eta_{b} \\
\eta^{\prime} & =\frac{1}{\sqrt{2}} \eta_{a}+\frac{1}{\sqrt{2}} \eta_{b}
\end{aligned}
$$

or

$$
\begin{gathered}
\eta_{a}=\frac{1}{\sqrt{2}} \eta+\frac{1}{\sqrt{2}} \eta^{\prime} \\
\eta_{b}=-\frac{1}{\sqrt{2}} \eta+\frac{1}{\sqrt{2}} \eta^{\prime},
\end{gathered}
$$

in complete analogy with the $K_{1}^{0}$ and $K_{2}^{0}$ states for the $K^{0}$ mesons. Assuming that

$$
M^{2}\left(\eta_{a}\right)=M^{2}\left(\eta_{b}\right)=\frac{1}{2}\left(M^{2}(\eta)+M^{2}\left(\eta^{\prime}\right)\right)
$$

and that the probabilities of the $\eta_{a}$ and $\eta_{b}$ production are equal, i.e. $\left\langle N\left(\eta_{a}\right)\right\rangle=$ $<N\left(\eta_{b}\right)>$, one obtains

$$
<N\left(\eta_{a}\right)>+<N\left(\eta_{b}\right)>=<N(\eta)>+<N\left(\eta^{\prime}\right)>
$$

and, consequently,

$$
<N\left(\eta_{a}\right)>=\frac{1}{2}\left(<N(\eta)>+<N\left(\eta^{\prime}\right)>\right) \text {. }
$$

These ideas lead to the modified adhoc isospin $I_{m}$ for the SU(3) pseudoscalar and vector meson nonets as shown in the following diagrams:

$$
\begin{aligned}
& K^{+} \quad K^{0} \quad \bar{K}^{0} \quad K^{-} \quad I_{m}=3 / 2 \quad K^{*+} \quad K^{* 0} \quad \bar{K}^{* 0} \quad K^{*-} \\
& \pi^{+} \quad \pi^{0} \quad \pi^{-} \quad I_{m}=1 \quad \rho^{+} \quad \rho^{0} \quad \rho^{-} \\
& \eta_{a} \quad \eta_{b} \quad I_{m}=1 / 2 \quad \omega_{a} \quad \omega_{b}
\end{aligned}
$$

Thus for the $\eta_{a}$, one has

$$
\left(2 I_{m}+1\right)<N\left(\eta_{a}\right)>=<N(\eta)>+<N\left(\eta^{\prime}\right)>,
$$

with $M^{2}\left(\eta_{a}\right)$ defined by (3). Similarly

$$
\left(2 I_{m}+1\right)<N\left(\omega_{a}\right)>=<N(\omega)>+<N(\phi)>,
$$

with

$$
M^{2}\left(\omega_{a}\right)=M^{2}\left(\omega_{b}\right)=\frac{1}{2}\left(M^{2}(\omega)+M^{2}(\phi)\right) .
$$

Then, the $(2 I+1) /(2 J+1)$ weight factor previously applied to the baryon production rates can be replaced by the $\left(2 I_{m}+1\right) /(2 J+1)$ factor, with the $I_{m}$ values for the $\mathrm{SU}(3)$ 
pseudoscalar and vector meson nonets given in the above diagram and with $I_{m}=I$ for the octet and decuplet baryons.

The resulting weighted production rates both for the mesons and baryons are shown in Fig. 3, where the rates measured simultaneously by the several LEP experiments are averaged. It shows that all data points, apart from the pions, lie on the same universal curve. In particular, the weighted production rates of $N, K^{*}(892)$ and $(\omega+\phi)$ with similar masses are practically the same. This implies that the production rates of the SU(3) pseudoscalar and vector mesons, octet and decuplet baryons/antibaryons are dominantly determined by their masses, spins and (modified) isospins.

The fit to the exponential $a \exp \left(-b M^{2}\right)$ (straight line in Fig. 3) yields the parameters

$$
a=11.28 \pm 0.31 \text { and } b=3.872 \pm 0.027\left(\mathrm{GeV} / c^{2}\right)^{-2},
$$

with $\chi^{2} / N D F=15 / 10$ (excluding the pion point).

In other words, the production rate of any particle considered above, besides the pions, with the given modified isospin projection and belonging to the pseudoscalar and vector meson nonets or to the octet and decuplet baryons, can be represented by a simple formula

$$
<N(\text { particle })>=\frac{2 J+1}{2 I_{m}+1} \cdot a \exp \left(-b M^{2}\right) .
$$

From (4) one can estimate the $\Delta^{++}(1232)$ production rate, not yet measured in the LEP experiments, $\left\langle N\left(\Delta^{++}\right)\right\rangle=0.032 \pm 0.001$. This estimate is from 2 to 3 times smaller than the corresponding values of $0.0945,0.0995$ and 0.0770 predicted by the Jetset, Herwig and UCLA models. Therefore, although the measurement of the $\Delta^{++}$ production is quite difficult (it is a relatively broad resonance sitting on top of a large combinatorial background and particle identification is essential for its detection), it will be quite interesting to check the prediction following from (4).

The measured $\Omega^{-}$production rate $[10]$ (see Table 1 ) is consistent with the trend of the data within the error, but nevertheless is higher than the fitted lines in Figs. 2 and 3. The expected value from (4) is $\left\langle N\left(\Omega^{-}\right)\right\rangle=0.00089 \pm 0.00003$. It is also of interest whether the new more precise measurements with higher statistics now accumulated by the LEP experiments will confirm this indication.

Obviously, one is tempted to ask what is the meaning of the parameters $a$ and $b$ in the expression (4) and how they depend on the center-of-mass energy? In order to answer this question, we repeated our analysis using the available data at PETRA and PEP energies. The measured meson and baryon production rates averaged over similar experiments were taken from the review [16]. The results are presented in Fig. 4. One sees that the data at $Z^{0}$ pole and at lower energies differ only in normalization (reflecting the rise of multiplicities with increasing energy). The result of the fit to the expression (4) (the $\Omega^{-}$and pions not included in the fit) are:

$$
a=8.00 \pm 0.35 \quad \text { and } \quad b=3.971 \pm 0.053\left(\mathrm{GeV} / c^{2}\right)^{-2}
$$

with $\chi^{2} / N D F=27 / 6$. Thus, the observed universal dependence of the particle production rates on their mass is even more striking, since even the value of the slope parameter can be fixed. Is it not surprising that knowing the slope value at PETRA and PEP energies one can predict the relative production rates of particles at LEP energies and vice versa? We are unable to find a plausible interpretation of the slope parameter value at the present stage. The values of $1 / \sqrt{b}$ are:

$$
1 / \sqrt{b}=508 \pm 2 \mathrm{MeV} / c^{2}
$$


for LEP data and

$$
1 / \sqrt{b}=502 \pm 4 \mathrm{MeV} / c^{2}
$$

for PETRA/PEP data. They are not far away from the $\mathrm{K}$ meson mass, but this can be a simple coincidence and should be verified using more precise LEP data soon available.

For the pions, the production rate is larger than expected from the trend of the other data. This is perhaps not so surprising and, in fact, much more interesting than if the pion rate agreed with the universal dependence observed for the other particles. First of all, the production of pions, more than the production of any other particles, is dominated by decays of numerous resonances. Not being able to separate the prompt particle production rates, one should be careful in interpreting the pion excess. On the other hand, the spin and isospin correction factors are the statistical factors which are expected to work not only for the prompt particle production, but also in their decays, if one considers all particle species. We should not also forget that the pions are Goldstone bosons. Therefore it can not be excluded that some part of them originates directly from the QCD vacuum.

In conclusion, we observe a striking regularity in meson and baryon production rates in $e^{+} e^{-}$annihilations at large energies. Contrary to a general belief and assumptions used in widely used models, the $2 J+1$ spin counting works. The production rates of all particles (apart from pions) belonging to the SU(3) nonets of the pseudoscalar and vector mesons and to the octet and decuplet of the baryons are determined only by particle spins, isospins and their masses. These production rates as a function of particle mass squared are described by a simple formula (4) with very close values of the parameter $b$ at LEP and PETRA/PEP energies.

\section{Acknowledgements}

We thank J. Ellis, A. A. Logunov, L. Montanet and T. Sjöstrand for interesting discussions and S. B. Chan and C. D. Buchanan for providing us with the numerical values of the UCLA predictions for the meson and baryon production rates. We are grateful to the DELPHI and L3 Collaborations for the permission to use some unpublished values of production rates. We are indebted to A. De Angelis, J.-E. Augustin, J. Guy and W. Venus for reading the manuscript and for the suggested improvements. 


\section{References}

[1] B. Andersson et al., Phys. Rep. 97 (1983) 31.

[2] G. Marchesini and B. R. Webber, Nucl.Phys. B238 (1984) 1.

[3] T. Sjöstrand, Comp. Phys. Comm. 39 (1986) 347; CERN preprint CERNTH.6488/92; T. Sjöstrand and M. Bengtsson, Comp. Phys. Comm. 43 (1987) 367.

[4] G. Marchesini et al., Comp. Phys. Comm. 67 (1992) 465.

[5] S. B. Chun and C. D. Buchanan, Phys. Lett. B308 (1993) 153 and private communication from S. B. Chun.

[6] P. V. Chliapnikov and V. A. Uvarov, Phys. Lett. B251 (1990) 192.

[7] DELPHI Collab., P. Abreu et al., Phys. Lett. B275 (1992) 231.

[8] DELPHI Collab., Strange Baryon Production in $Z^{0}$ Hadronic Decays, paper presented to the 27th Int. Conf. on High Energy Physics, Glasgow, 1994.

[9] OPAL Collab., P. D. Acton et al., Phys. Lett. B291 (1992) 503.

[10] OPAL Collab., Strange Baryon Production and Correlations in Hadronic $Z^{0}$ Decays, paper presented to the 27th Int. Conf. on High Energy Physics, Glasgow, 1994.

[11] L3 Collab., M. Acciari et al., Phys. Lett. B328 (1994) 223.

[12] ALEPH Collab., D. Buskulic et al., Production of $\mathrm{K}^{0}$ and $\Lambda$ in Hadronic $Z^{0}$ decays, preprint CERN-PPE/94-74 (1994) (to appear in Z. Phys. C).

[13] DELPHI Collab., S. Plaszynski and P. Roudeau, DELPHI Note 94-135 PHYS 445 (1994) (unpublished).

[14] OPAL Collab., R. Akers et al., Z. Phys. C63 (1994) 181.

[15] DELPHI Collab., Inclusive $\mathrm{K}^{ \pm}, p / \bar{p}$ Production in $Z^{0}$ Decays Measured with the DELPHI Detector, paper presented to the 27th Int. Conf. on High Energy Physics, Glasgow, 1994.

[16] A. De Angelis, J. Physics G19 (1993) 1233.

[17] B. Andersson, G. Gustafson and T. Sjöstrand, Physica Scripta B32 (1985) 574.

[18] OPAL Collab., P. D. Acton et al., Phys. Lett. B305 (1993) 415.

[19] DELPHI Collab., P. Abreu et al., Phys. Lett. B318 (1993) 249.

[20] Particle Data Group, M. Aguilar-Benitez et al., Phys. Rev., D50 (1994) 1173.

[21] DELPHI Collab., P. Abreu et al., Production Characteristics of $\mathrm{K}^{0}$ and Light Meson Resonances in Hadronic Decays of the $Z^{0}$, preprint CERN-PPE/94-130 (1994) (to appear in Z. Phys. C).

[22] OPAL Collab., G. Alexander et al., Phys. Lett. B264 (1991) 467.

[23] OPAL Collab., P. D. Acton et al., Phys. Lett. B305 (1993) 407.

[24] DELPHI Collab., P. Abreu et al., Phys. Lett. B298 (1992) 236.

[25] OPAL Collab., Inclusive Strange Vector and Tensor meson Production in Hadronic $Z^{0}$ Decays, paper presented to the 27th Int. Conf. on High Energy Physics, Glasgow, 1994.

[26] ALEPH Collab., D. Buskulic et al., Phys. Lett. B292 (1992) 210.

[27] L3 Collab., Yi-Jin Pei, Studies of meson production in $Z^{0}$ decays, CERN Seminar, 7 September 1994 (unpublished).

[28] DELPHI Collab., A. De Angelis, F. Scuri and L. Vitale, Inclusive Production of the $\phi(1020)$ in the Hadronic Decays of the $Z^{0}$, DELPHI Note 93-24 PHYS 270 (1993) (unpublished). 
Table 1: The total mean baryon and meson multiplicity per hadronic $Z^{0}$ decay, $<N(\exp )$.$\rangle , measured in LEP experiments in comparison with the Jetset 7.4 PS,$ Herwig 5.8 and UCLA 7.44 models.

\begin{tabular}{|c|c|c|c|c|c|}
\hline Particle & $<N(\exp )>$. & Experiment & Jetset & Herwig & UCLA \\
\hline$p$ & $0.460 \pm 0.055$ & OPAL [14] & 0.598 & 0.473 & 0.590 \\
\hline$\Lambda$ & $0.193 \pm 0.008$ & ALEPH [12] & 0.193 & 0.216 & 0.182 \\
\hline$\Lambda$ & $0.179 \pm 0.0085$ & DELPHI [8] & & & \\
\hline$\Lambda$ & $0.185 \pm 0.020$ & L3 [11] & & & \\
\hline$\Lambda$ & $0.182 \pm 0.011$ & OPAL $[10]$ & & & \\
\hline$\Sigma^{+}$ & $0.0425 \pm 0.0090$ & DELPHI [13] & 0.0350 & 0.0360 & 0.0323 \\
\hline$\Xi^{-}$ & $0.0125 \pm 0.0011$ & DELPHI [8] & 0.0137 & 0.0283 & 0.0114 \\
\hline$\Xi^{-}$ & $0.0120 \pm 0.0009$ & OPAL $[10]$ & & & \\
\hline$\Sigma^{+}(1385)$ & $0.00955 \pm 0.00133$ & DELPHI [8] & 0.0186 & 0.0363 & 0.0205 \\
\hline$\Sigma^{+}(1385)$ & $0.00930 \pm 0.00125$ & OPAL $[10]$ & & & \\
\hline$\Xi^{0}(1530)$ & $0.00305 \pm 0.00065$ & DELPHI [8] & 0.0027 & 0.0136 & 0.0039 \\
\hline$\Xi^{0}(1530)$ & $0.00360 \pm 0.00065$ & OPAL $[10]$ & & & \\
\hline$\Omega^{-}$ & $0.00140 \pm 0.00045$ & OPAL [10] & 0.00035 & 0.00362 & 0.00055 \\
\hline$\pi^{+}$ & $8.53 \pm 0.22$ & OPAL [14] & 8.48 & 8.82 & 8.38 \\
\hline$\pi^{0}$ & $9.18 \pm 0.73$ & L3 [11] & 9.60 & 9.82 & 9.51 \\
\hline$K^{0}$ & $1.031 \pm 0.024$ & ALEPH [12] & 1.11 & 1.19 & 0.99 \\
\hline$K^{0}$ & $0.981 \pm 0.030$ & DELPHI [21] & & & \\
\hline$K^{0}$ & $1.02 \pm 0.07$ & L3 [11] & & & \\
\hline$K^{0}$ & $1.05 \pm 0.07$ & OPAL [22] & & & \\
\hline$K^{+}$ & $1.13 \pm 0.08$ & DELPHI [15] & 1.15 & 1.21 & 1.09 \\
\hline$K^{+}$ & $1.21 \pm 0.065$ & OPAL $[14]$ & & & \\
\hline$\eta$ & $0.90 \pm 0.10$ & L3 [11] & 1.00 & 1.02 & 0.779 \\
\hline$\eta$ & $0.97 \pm 0.12$ & ALEPH [26] & & & \\
\hline$\eta^{\prime}$ & $0.13 \pm 0.05$ & ALEPH [26] & 0.298 & 0.141 & 0.154 \\
\hline$K^{*+}(890)$ & $0.36 \pm 0.04$ & OPAL $[23]$ & 0.555 & 0.403 & 0.387 \\
\hline$K^{*+}(890)$ & $0.356 \pm 0.034$ & DELPHI [21] & & & \\
\hline$K^{* 0}(890)$ & $0.485 \pm 0.180$ & DELPHI [24] & 0.550 & 0.403 & 0.355 \\
\hline$K^{* 0}(890)$ & $0.370 \pm 0.025$ & OPAL $[25]$ & & & \\
\hline$\rho^{0}$ & $1.21 \pm 0.15$ & DELPHI [21] & 1.51 & 1.32 & 1.14 \\
\hline$\omega$ & $1.13 \pm 0.18$ & L3 $[27]$ & 1.35 & 0.858 & 1.00 \\
\hline$\phi$ & $0.100 \pm 0.008$ & OPAL $[25]$ & 0.194 & 0.121 & 0.124 \\
\hline$\phi$ & $0.097 \pm 0.014$ & DELPHI [28] & & & \\
\hline
\end{tabular}




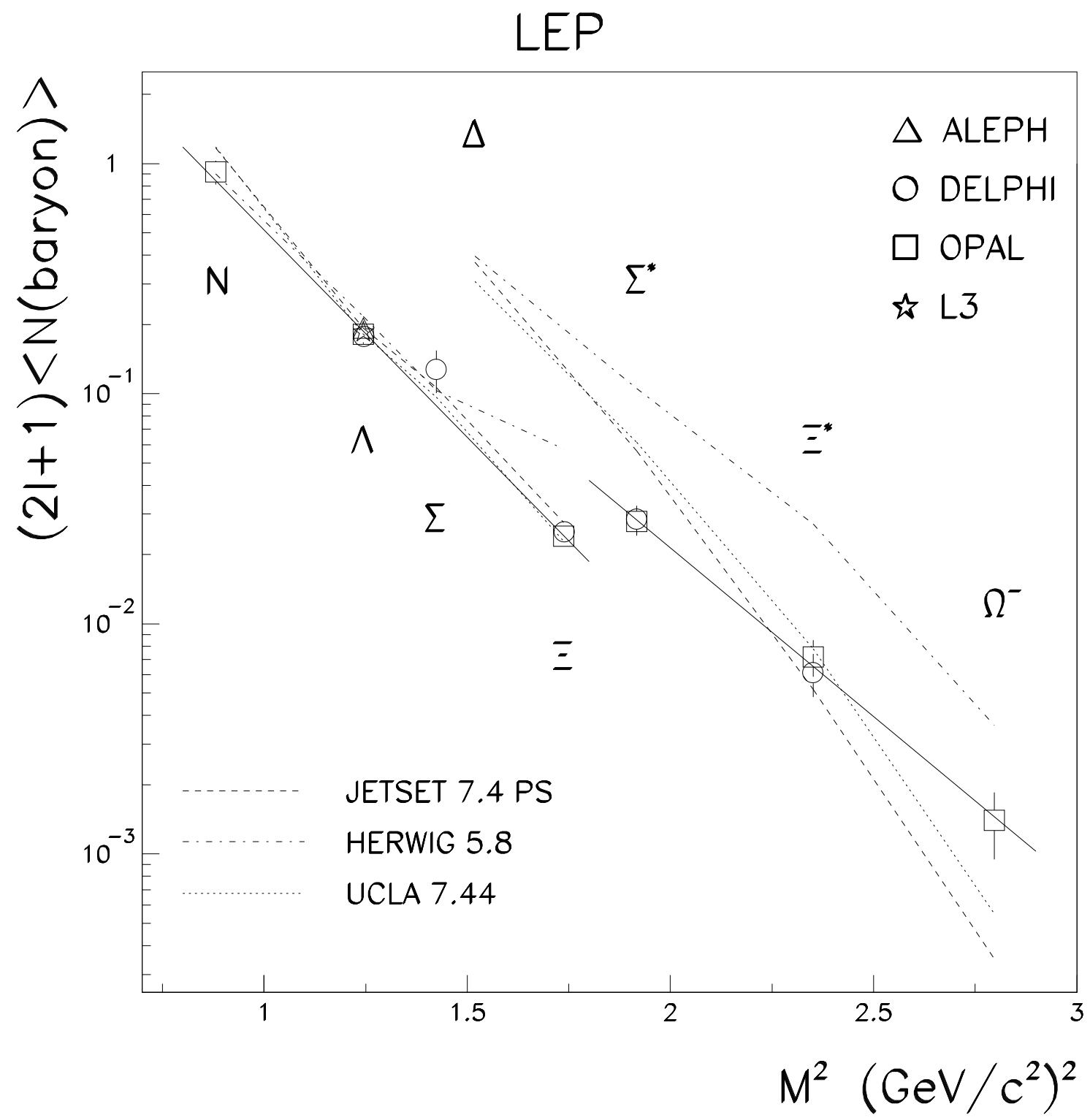

Figure 1: Production rates of octet and decuplet baryons at $Z^{0}$ pole at LEP, weighted with the $2 I+1$ isospin counting factor, as a function of particle mass squared. The solid lines show the results of the fits to the form $a \exp \left(-b M^{2}\right)$ described in the text. Predictions of the Jetset, Herwig and UCLA models with default parameters are also shown. 


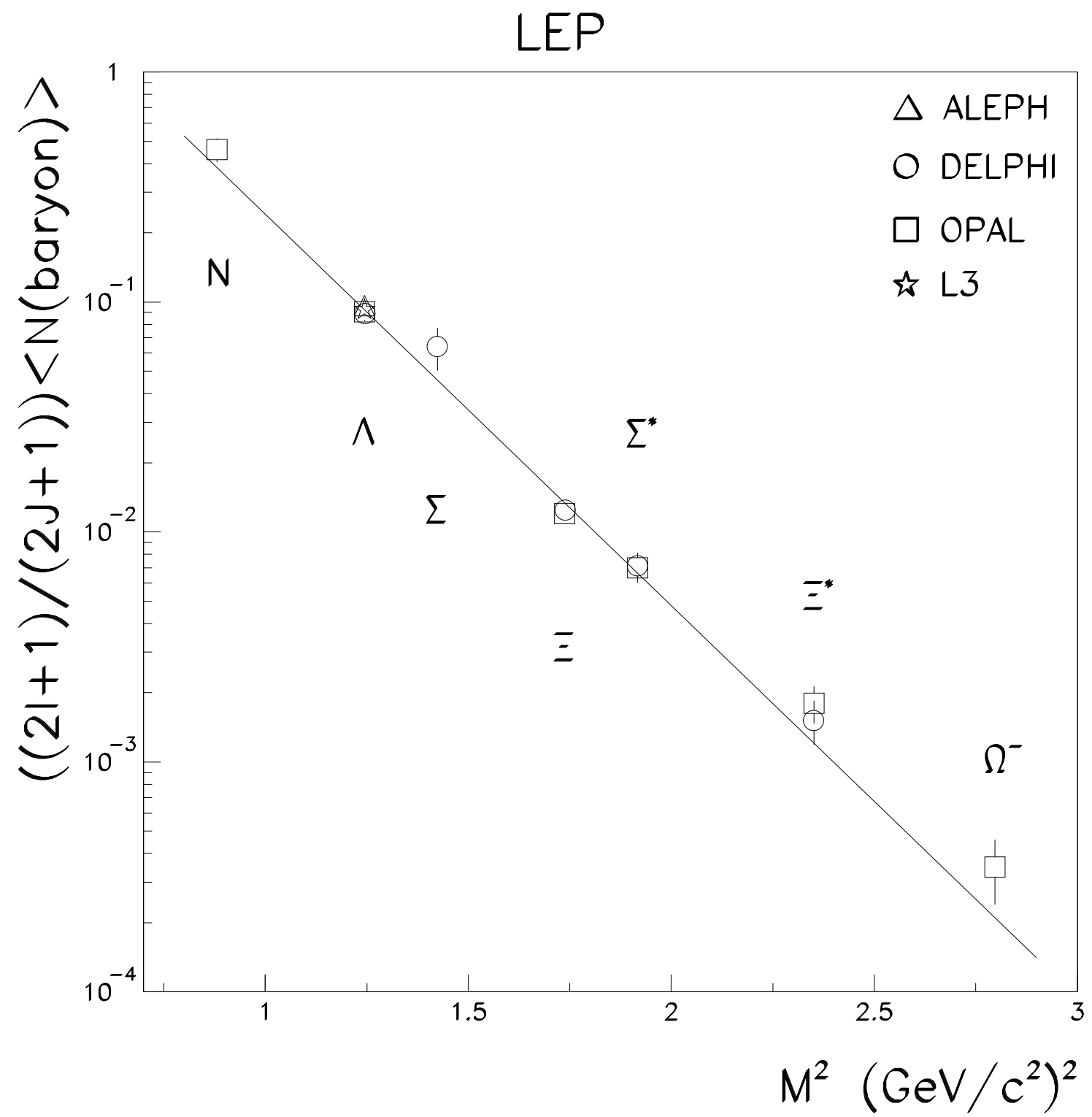

Figure 2: Production rates of octet and decuplet baryons at $Z^{0}$ pole at LEP, weighted with the $2 I+1$ isospin and $1 /(2 J+1)$ spin counting factors, as a function of particle mass squared. The line shows the result of the fit to the form $a \exp \left(-b M^{2}\right)$ described in the text. 
LEP

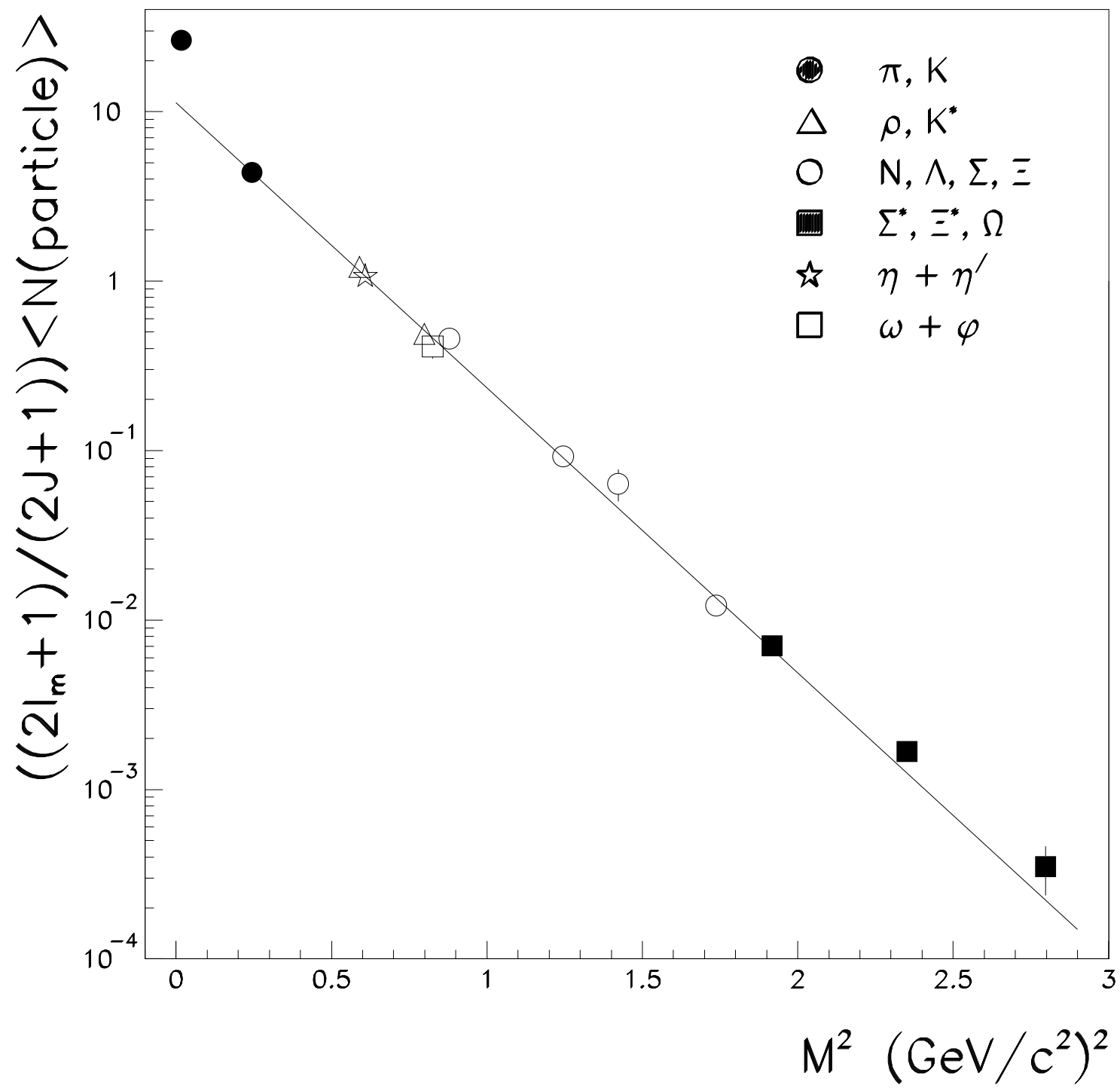

Figure 3: Production rates of pseudoscalar and vector mesons, and octet and decuplet baryons at $Z^{0}$ pole at LEP, weighted with the $\left(2 I_{m}+1\right) /(2 J+1)$ factor, as a function of particle mass squared. The line shows the result of the fit to the form $a \exp \left(-b M^{2}\right)$ described in the text. 
PEP, PETRA

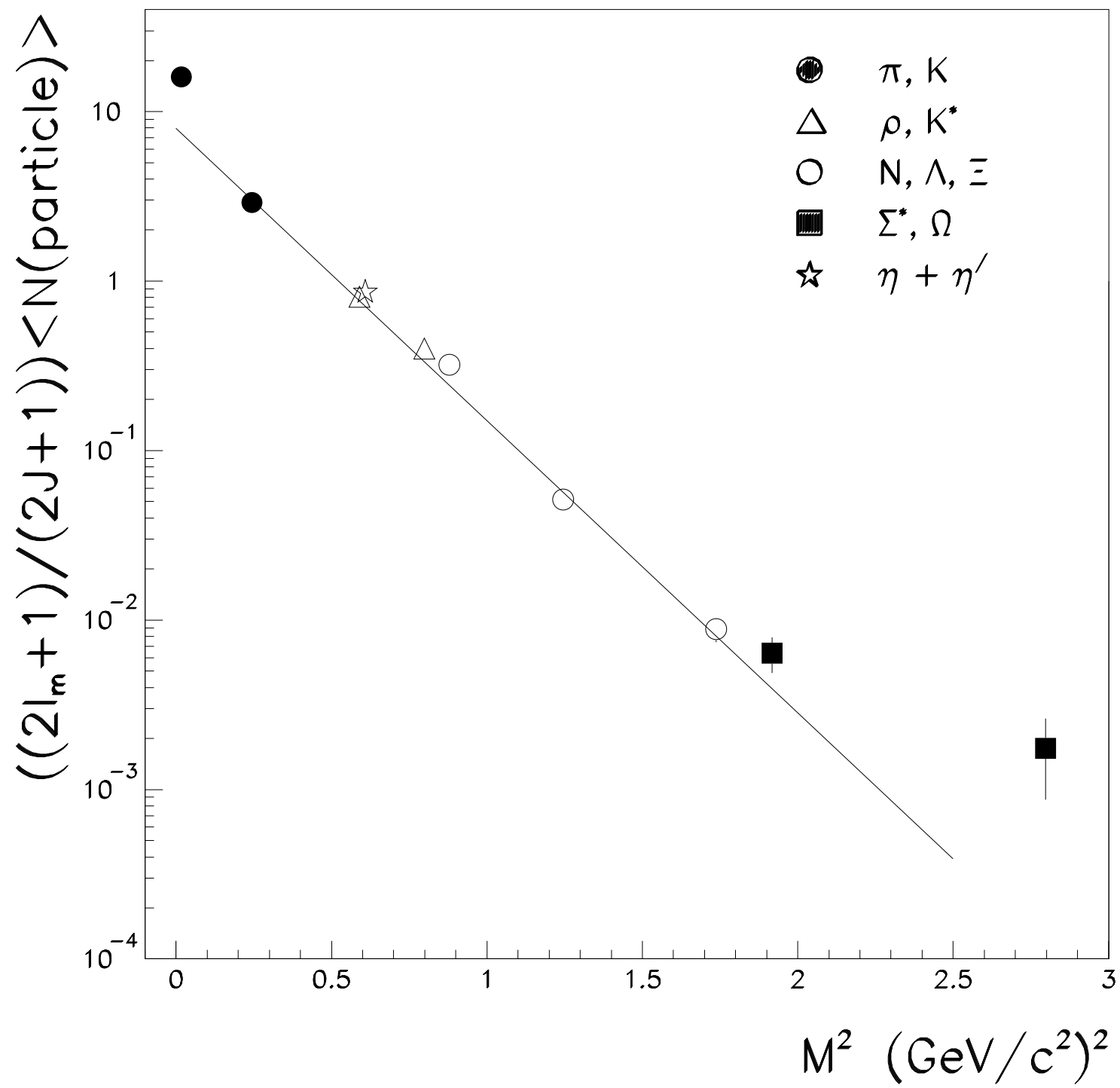

Figure 4: Production rates of pseudoscalar and vector mesons, and octet and decuplet baryons at PETRA and PEP weighted with the $\left(2 I_{m}+1\right) /(2 J+1)$ factor, as a function of particle mass squared. The line shows the result of the fit to the form $a \exp \left(-b M^{2}\right)$ described in the text. 